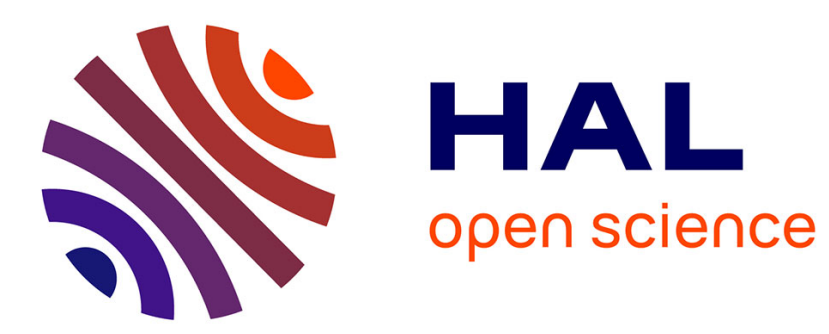

\title{
Robust multi-objective and multi-level optimization of complex mechanical structures
}

\author{
S Ghanmi, M Guedri, M L Bouazizi, Noureddine Bouhaddi
}

\section{To cite this version:}

S Ghanmi, M Guedri, M L Bouazizi, Noureddine Bouhaddi. Robust multi-objective and multi-level optimization of complex mechanical structures. Mechanical Systems and Signal Processing, 2011, 25

(7), pp.2444 - 2461. 10.1016/j.ymssp.2011.02.011 . hal-01512525

\section{HAL Id: hal-01512525 \\ https://hal.science/hal-01512525}

Submitted on 23 Apr 2017

HAL is a multi-disciplinary open access archive for the deposit and dissemination of scientific research documents, whether they are published or not. The documents may come from teaching and research institutions in France or abroad, or from public or private research centers.
L'archive ouverte pluridisciplinaire HAL, est destinée au dépôt et à la diffusion de documents scientifiques de niveau recherche, publiés ou non, émanant des établissements d'enseignement et de recherche français ou étrangers, des laboratoires publics ou privés.

\section{(c)(1)}

Distributed under a Creative Commons Attribution| 4.0 International License 


\title{
Robust multi-objective and multi-level optimization of complex mechanical structures
}

\author{
S. Ghanmi ${ }^{\text {a }}$, M. Guedri ${ }^{\text {a }}$, M.-L. Bouazizi ${ }^{\text {a }}$, N. Bouhaddi ${ }^{\text {b }}$ \\ a Preparatory Engineering Institute of Nabeul (IPEIN), 8000 M'rezgua, Nabeul, Tunisia \\ ${ }^{\mathrm{b}}$ FEMTO-ST Institute UMR 6174-Applied Mechanics Laboratory R. Chaléat, University of Franche-Comté, 24 Chemin de l'Epitaphe, 25000 Besançon, France
}

\begin{abstract}
This paper presents a new approach to robust multi-objective and multi-level optimization of the design of complex mechanical structures. The optimization is at two levels: system and elements. At system-level, the robust multi-objective problem has four cost functions: on the one hand the minimization of the global mass and displacement at a fixed point of the mechanical structure, and on the other hand the maximization of both the robustness and the displacement of the mass. At elementlevel the robust multi-objective problem has two cost functions: minimization of the element mass and maximization of its robustness. A robust condensation technique, based on an enriched Karhunen-Loève condensation, is used for complex structures which require a large finite element model. In contrast to existing formulations, this new approach takes into account uncertainties in the design parameters at system-level and element-level. It also allows for the task sharing which is commonly used in structural engineering.
\end{abstract}

Keywords:

Multi-objective optimization

Multi-level optimization

Uncertainties

Genetic algorithm

Robustness

Karhunen-Loève transformation

\section{Introduction}

Nowadays, the design of complex structures (which require a large finite element model (FE model) such as aircraft, aerospace, etc., require the use of optimization methods. The size of the optimization problem depends on the number of variables and constraints required, especially for the design of structures with large interactions between their various components. Treating the problem in the conventional manner with all components considered simultaneously leads to excessive computer run times (CPU times). In order to control this growth in the size of the problem, a multi-level approach has to be used to decompose the problem (system-level) down into several sub-problems (element-level) taking into account the structural coupling effects. Such an approach enables us to use parallel computer processing, leading to a significant reduction in the computer run time and a decrease in the time needed the design cycle.

A multi-level approach is needed to decompose the entire system problem (system-level) into several sub-problems (element-level) taking into account the various coupling effects. The multi-level approach allows individual system components to be optimized, thus significantly reducing the time needed for the computation and the design cycle. Various concepts have been proposed [1-7] to decompose large optimization problems down into sub-problems. It should be noted that the design parameters of the global structure affect both the structure and sub-structures (density, Young modulus, etc.), while the design parameters of the sub-structures can be local dimensions (e.g. geometric dimensions: thickness, length, width). The constraints on the structure are global (e.g. structural displacement), while the constraints 
on the sub-structures are local (e.g. local stress or strain). The multi-level optimization proceeds by iterating in both directions between the solution of the structure and the sub-structures.

The use of multi-objective and multi-level optimization [1-7] usually faces the following problems:

- The multi-objective optimization problem is transformed into a single-objective problem. This transformation requires a priori knowledge of the weight of each cost function in relation to the others. In practice this is not always easy. There may be a lack of experience or information, or the cost functions are not compatible. In addition, if the design space is not convex, the weighting method will ignore the convex part of the whole Pareto optimal solutions, whereas this is the part of solution where a compromise is to be found.

- Earlier authors [1-7] did not take the uncertainties in the design parameter into account during optimization. Indeed, it is well known that a good theoretical solution can be catastrophic in practice if, for example, the errors during manufacturing mean that the design variables are not achieved with sufficient precision. A solution which is suboptimal but is stable with regard to the uncertainty will be of much greater use to the designer.

Several optimization algorithms have been used to solve the multi-objective and multi-level problems. Evolutionary algorithms like the genetic algorithm (GA) [1] or gradient [2] have been used at either system-level or element-level, or specific computer codes such as AISC-LRFD, AISC and GBJ17-88 have been used [3] to optimize the steel frames.

This paper presents an original contribution to multi-objective and multi-level optimization. Our proposed method takes into account the uncertainties in the design parameters at system-level and element-level. Taking these uncertainties into account in the multi-objective optimization has required the introduction of robust solutions to these uncertainties. Our solution uses a multi-level GA. The number of useful GAs depends on the number of elements at each level of optimization.

In order to further reduce the computing time and ensure a good prediction by the FE models, at either the system-level or element-level, one can couple the stochastic finite element method (SFEM) with a robust dynamic condensation method with respect to the stochastic parametric modifications [8,9] in order to construct a robust reduced uncertainty model. This condensation method is based on the Karhunen-Loeve (KL) approach. This method permits us to assign specific uncertainties (weak, means or large level) to zones or sub-structures. The extension of this robust condensation method to complex structures which need a large FE model also allows us to obtain a fast, robust, modal synthesis for such structures.

This paper is structured as follows: the robust multi-objective and multi-level optimization approach is presented in Section 2. The multi-level approach for beam and plate structure models is considered in Section 3.

\section{Proposed robust multi-objective and multi-level optimization strategy}

This section is divided into four sub-sections. First subsection gives the principle of multi-objective optimization, the second shows the robustness criterion used in the optimization procedure; the third presents the proposed formulation to the multi-level decomposition, while the fourth subsection is reserved to method of robust reduction of FE model of mechanical structures to be studied.

\subsection{Robust multi-objective optimization}

Before discussing robust design optimization, the mathematical statement of a deterministic (nominal) design optimization problem is needed

$$
\left\{\begin{array}{l}
\min F(x)=\left(f_{1}(x), f_{2}(x), \ldots, f_{k}(x)\right)^{T} \\
x \in S
\end{array}\right.
$$

where $f_{1}(x), f_{2}(x), \ldots, f_{k}(x)$ are cost functions with $k \geq 2 ;\left(x_{1}, x_{2}, \ldots x_{n}\right)^{T}$ is the vector of " $n$ " optimization parameters; $S \in R^{n}$ represents all of the realizable solutions; and $F(x)=f_{1}(x), f_{2}(x), \ldots, f_{k}(x)$ is the vector of the functions to be optimized.

For a practical design problem, $F$ is non-linear multi-modal and not necessarily analytical. We now consider the robustness of the objective functions.

The design variable $x_{i}(1 \leq i \leq n)$ is assumed to be normally distributed with the mean, $\mu_{x_{i}}$ and the standard deviation, $\sigma_{x_{i}}$. The tolerance of a design variable, $\Delta x_{i}$ is equal to $3 \sigma_{x_{i}}$ thus, $99.73 \%$ of the design variables are between $\Delta x_{i}-3 \sigma_{x_{i}}$ and $\Delta x_{i}+3 \sigma_{x_{i}}$. Our proposed method aims to obtain the robust Pareto front when the design variables vary within this tolerance interval.

The distribution of the objective function is a consequence of the distribution of the design variables. The tight dispersal of the objective function reduces the manufacturing cost since the tolerance bands of the design variables can be loosened. Classically, the optimal solution robustness is evaluated from the deterministic optimization procedure. Kwon-Lee [10,11] has proposed a robust optimization method in which a multi-objective function is defined to have the mean value and standard deviation of the original objective function. The principal disadvantage of this method is that it uses a weighting method. The weakness of the weighting method is that, unless the problem is convex, not all of the Pareto optimal solutions may be found. 
The robust multi-objective optimization (RMO) problem defined by Kwon-Lee $[10,11]$ is as follows:

$$
\left\{\begin{array}{l}
\underset{x}{\operatorname{minf}}(x)=\alpha \frac{\mu_{f}}{\mu_{f}^{*}}+(1-\alpha) \frac{\sigma_{f}}{\sigma_{f}^{*}} ; \quad 0 \leq \alpha \leq 1 \\
x: \text { random design }
\end{array}\right.
$$

where $\mu_{f}$ and $\sigma_{f}$ are the mean value and the standard deviation of the objective function; and $\mu_{f}^{*}$ and $\sigma_{f}^{*}$ are the optimal values, considering only the mean and the standard deviation, respectively. But in this case only non-convex admissible solutions were explored and optimal solutions could not be found.

The idea exploited in this paper is different. The robustness functions are introduced as an additional objective cost in the multi-objective optimization problem. The aim is to find both optimal and robust solutions. A RMO problem is defined as follows [12]:

$$
\left\{\begin{array}{l}
\min F(x)=\left(f_{1}(x), f_{1}^{v}(x), \ldots, f_{k}(x), f_{k}^{v}(x)\right)^{\mathrm{T}} \\
x \in S
\end{array}\right.
$$

where

$$
f_{i}^{v}(x)=\left(\sigma_{f_{i}} / \mu_{f_{i}}\right)=\frac{1}{f_{i}^{r}(x)}
$$

where $f_{i}^{v}(x)$ and $f_{i}^{r}(x)$ are, respectively, the vulnerability and robustness functions of the cost function $f_{i}(x)$ given by Eq. (4). The ratio $\sigma_{f_{i}} / \mu_{f_{i}}$ is the dispersion (or the vulnerability function), where $\mu_{f_{i}}$ and $\sigma_{f_{i}}$ are, respectively, the mean value and standard deviation of $f_{i}(x)$. We used the Latin Hypercube simulation [13] to evaluate the robustness functions with 1000 samples.

The robust solutions with respect to uncertainties are those that make it possible to simultaneously minimize the original objective functions $\left(f_{1}(x), f_{2}(x), \ldots, f_{k}(x)\right)$ and maximize their robustness $\left(f_{1}^{r}(x), f_{2}^{r}(x), \ldots, f_{k}^{r}(x)\right.$ ) (or to minimize their vulnerabilities $\left.\left(f_{1}^{v}(x), f_{2}^{v}(x), \ldots, f_{k}^{v}(x)\right)\right)$.

To search for robust and optimal solutions, one can exploit non-dominated sorting GAs of NSGA type (Non-dominated Sorting Genetic Algorithm) [14], which enable us to further explore the design space and to cover the entire Pareto optimal front.

The set of Pareto optimal solutions is that set formed by the solutions which are not dominated by other solutions. This set forms the first Pareto front. That is to say, if one considers a minimization problem, where $y, z \in S$ are two vectors of $\mathrm{n}$ optimization parameters, $y$ dominates $z(y>z)$ if and only if $\forall i \in[1, \ldots, n]: f_{i}(y) \leq f_{i}(z)$ and $\exists j \in[1, \ldots, n]: f_{j}(y)<f_{j}(z)$.

To illustrate the advantage of the proposed robustness criterion, one can consider a single-objective problem, and seeks a solution, which is both optimal and robust. The single-objective problem is to be optimized according to

$$
\left\{\begin{array}{l}
\min f(x)=2-e^{-\left(\frac{x-0.2}{0.004}\right)^{2}}-0.8 e^{-\left(\frac{x-0.6}{0.4}\right)^{2}} \\
x \in[0,1]
\end{array}\right.
$$

where $f(x)$ is the cost function to be minimized and " $x$ " is a distributed normal random variable. The multi-objective optimization problem is defined as

$$
\left\{\begin{array}{l}
\min _{x}\left(f(x), f^{v}(x)\right) \\
x \in[0,1]
\end{array}\right.
$$

The proposed robust optimization method is used to find the Pareto optimal design. Various tests allow us to define the parameters of our evolutionary algorithm: the population size (30), the total number of generations (50), the crossover probability $(0.8)$, the mutation probability $(0.1)$, and the sharing coefficient $\left(\sigma_{\text {share }}=0.2\right)$.

Figs. 1 and 2 show, respectively, the optimal solutions and the robust and optimal Pareto front resulting from the RMO procedure. All the optimal solutions $\left(x_{1}=0.2\right.$ and $\left.x_{2}=0.4\right)$ are found in this front. The designer is able to choose the robust optimal solutions that have the best rank in terms of vulnerability (Table 1 ).

To validate this strategy, we compared the solutions obtained by their deterministic and robust classifications. For the deterministic optimization procedure, the global solution is " $x_{1}$ ", which represents the optimum solution (ignoring the robustness), while for the robust optimization procedure, " $x$ " is the robust optimum solution with low vulnerability. The deterministic optimum performs better than the robust optimum.

The results show that the deterministic optimization is insufficient and non-robust because it does not take into account the level of the cost functions, and the effect of uncertainties on the design parameters. Our proposed approach to solving the robust optimization problem, based on the introduction of a dispersion function with respect to uncertainties, makes it possible to find a solution that is both optimal and robust.

\subsection{Multi-level optimization}

The robust multi-objective and multi-level optimization (RMOL) problem is set as follows: we consider a complex mechanical structure consisting of an assembly of $K$ elements. The detailed geometry of the element " $i$ " is defined by the vector of the local 


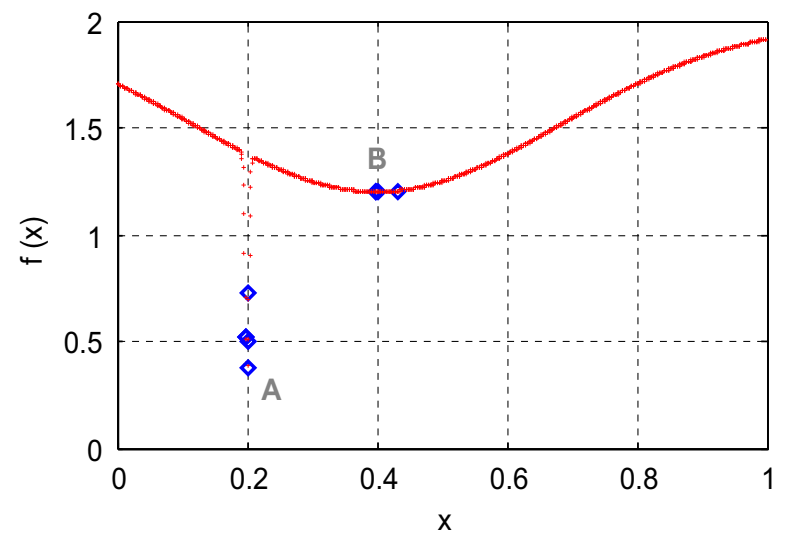

Fig. 1. Optimal solutions: A—Global optimum and B-Local optimum.

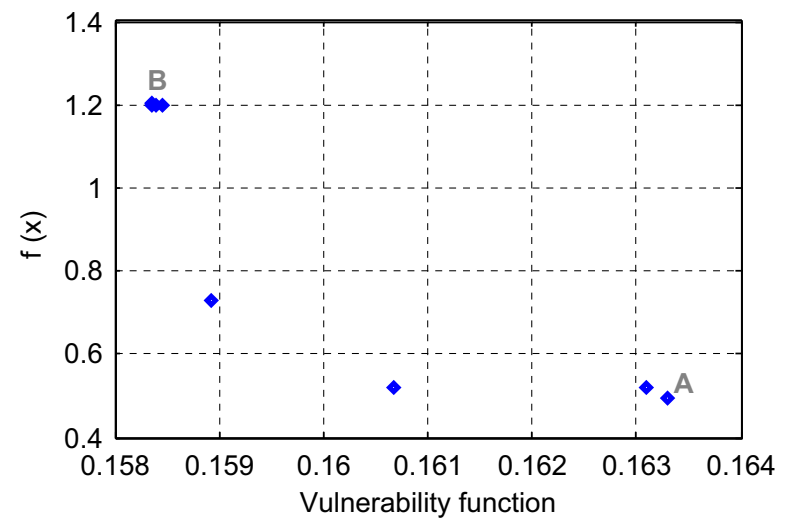

Fig. 2. Robust and optimal Pareto front: A—Global but non-robust optimum and B_Local robust optimum.

Table 1

Comparison between deterministic and robust solutions.

\begin{tabular}{lllll}
\hline Solution & Variable “ $x$ ” & $f(x)$ & $f^{\prime}(x)$ & Deterministic rank \\
\hline A & 0.2 & 0.496 & 0.163 & 1 \\
B & 0.4 & 1.2 & 0.158 & 2 \\
\hline
\end{tabular}

variables associated with $X=X_{i / i=1 \ldots K}=\left(x_{i, j}\right)_{j=1, \ldots, n l_{i}}$ and the associated global variables, $Y=Y_{i / i}=1 \ldots K=\left(y_{i, j}\right)_{j=1, \ldots, n g_{i}}$. In addition, there is a function $g: \mathfrak{R}^{n l_{i}} \rightarrow \mathfrak{R}^{n g_{i}}$ such that: $Y_{i}=g\left(X_{i}\right)$.

For system-level optimization, the RMO problem is defined as follows:

$$
\left\{\begin{aligned}
& \min F(Y)=\left(f_{1}(Y), f_{1}^{v}(Y), \ldots, f_{m}(Y), f_{m}^{v}(Y)\right)^{T} \\
& \text { subject to: } G_{j}(Y) \leq 0 ; \quad j=1, \ldots, n_{s} \\
& Y_{\min } \leq Y \leq Y_{\operatorname{Max}}
\end{aligned}\right.
$$

where $f_{i / i=1, \ldots, m}$ and $f_{i / i=1, \ldots, m}^{v}$ are, respectively, the cost functions and their vulnerability, and $G_{j}$ represents the constraints of the system-level problem.

For the element-level optimization (for the $i$ th element), the RMO problem is defined as follows:

$$
\left\{\begin{aligned}
\min F_{i}(X)= & \left(f_{i_{1}}(X), f_{i_{1}}^{v}(X), \ldots, f_{i_{m}}(X), f_{i_{m}}^{v}(X)\right)^{T} \\
\text { subject to: } & G_{i}\left(Y^{*}, X\right) \leq 0 \\
& G_{j}(X) \leq 0 ; \quad j=1, \ldots, n_{i} \\
& X_{\min } \leq X \leq X_{\operatorname{Max}}
\end{aligned}\right.
$$




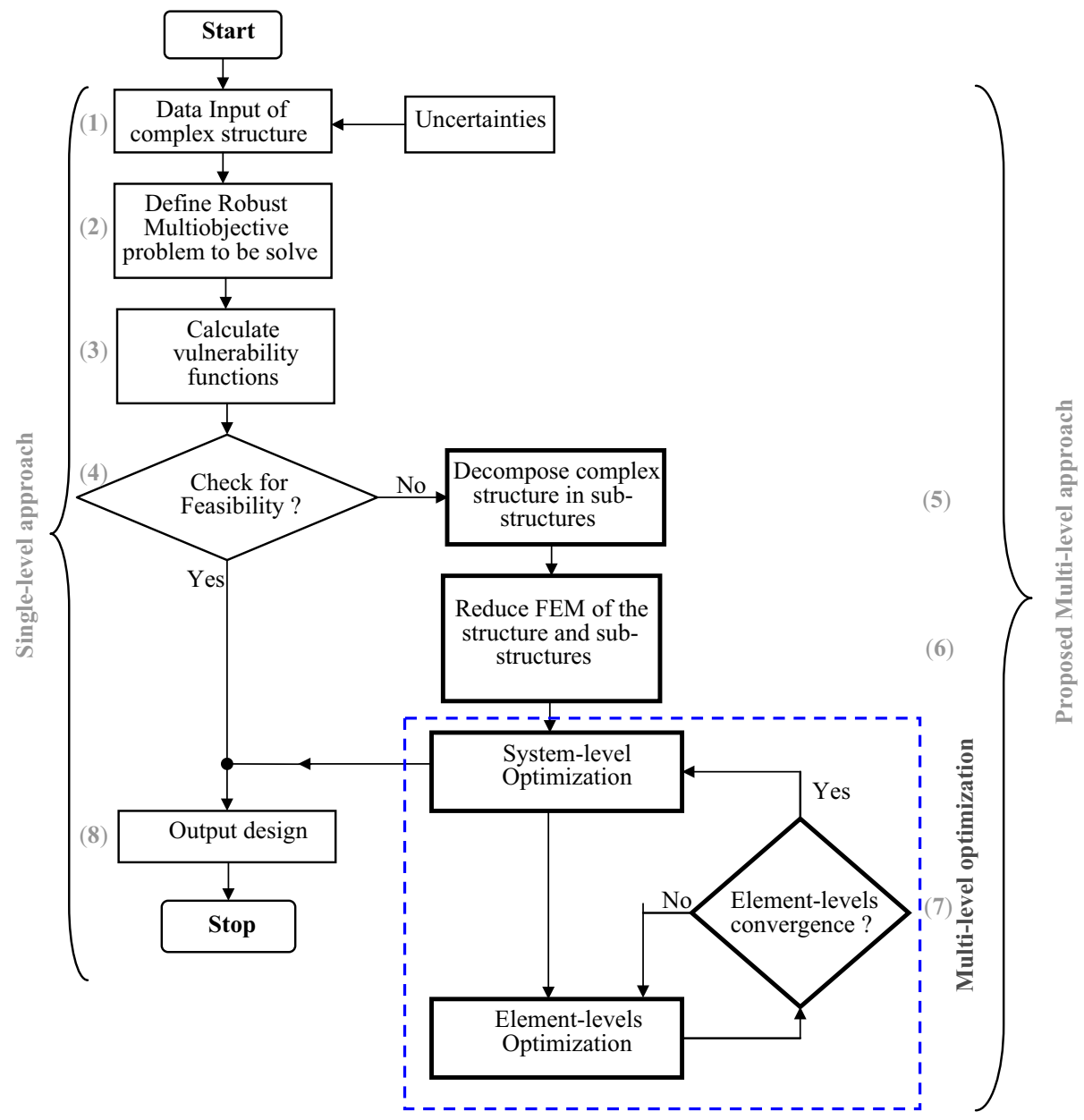

Fig. 3. Flowchart of the optimization procedure.

where $F_{i}(X)$ is the vector of the cost functions and their vulnerabilities of the element $i, G_{j}(X)$ are the constraints of the local problem, and $n_{i}$ is the number of constraints; and $G_{i}\left(Y^{*}, X\right)$ is the additional constraint of equality. This constraint links design variables at different levels and simplifies the coupling between them. $\left({ }^{*}\right)$ denotes the variables, whose values are the optimum outputs at element-levels and they are considered as fixed inputs in phase of optimization of system-level.

Fig. 3 presents a flowchart of the proposed optimization procedure. This proposed optimization with respect to the multi-level decomposition is specified by the following steps:

Step 1. Define uncertain design parameters.

Step 2. Define robust multi-objective problem to be solved.

Step 3. Calculate vulnerability functions of each cost function: this is done by determining the mean value and standard deviation of each function.

Step 4. Perform feasibility test:

This test depends on:

- Type of evolutionary algorithm used for optimization (GA...).

- Complexity of model.

- Number of design parameters.

- Correlations between design parameters.

- Complexity of cost functions to be solved (non-linear, multi-physics).

If answer is positive, go to Step 8 .

If answer is negative, go to Step 5.

Step 5. Decompose structure into sub-structures: decomposition depends on geometry (section, length,), mechanical properties (Young's modulus, density), mechanical stress (bending, compression ...), etc.

Step 6. Reduce FE model of structure and sub-structures by KL approach. 


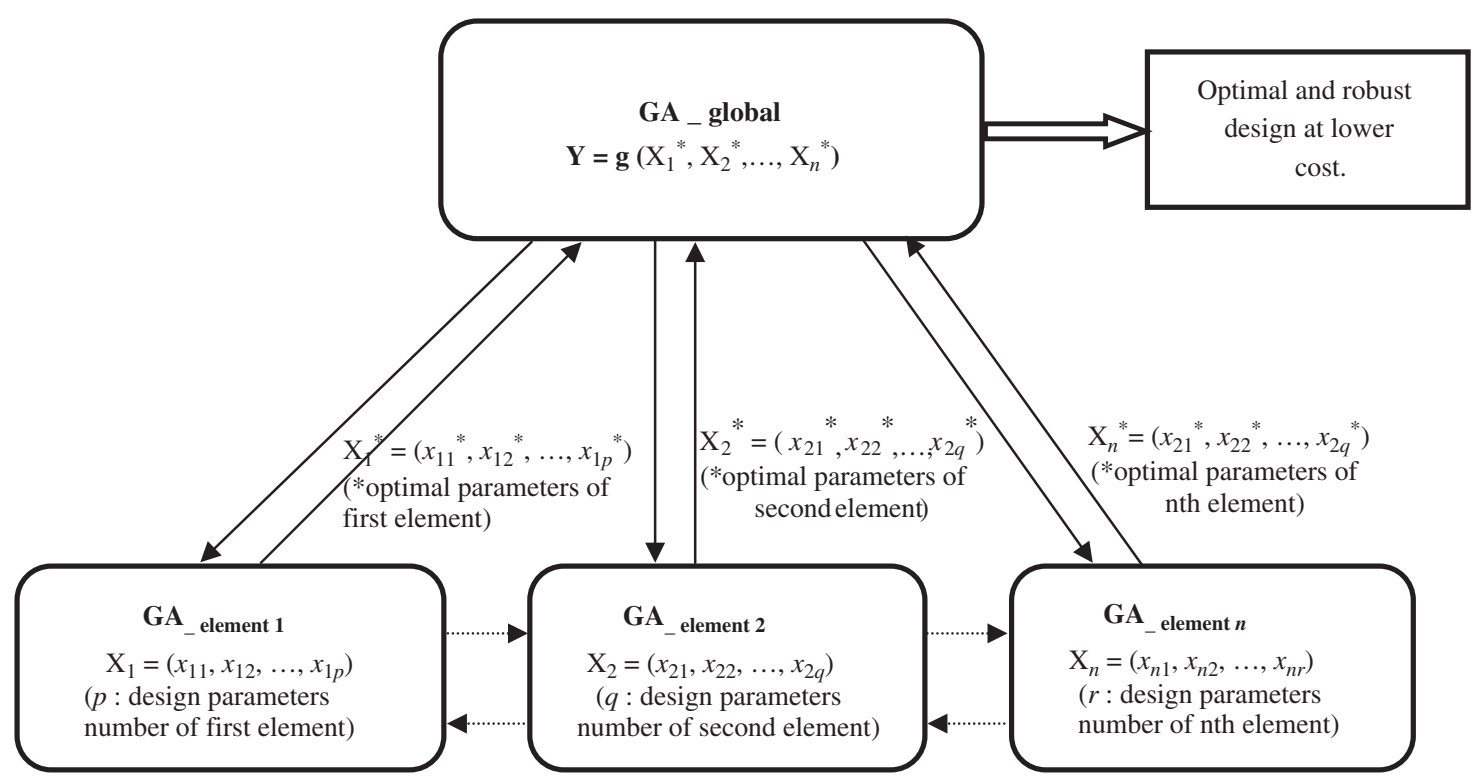

Fig. 4. Multi-level algorithm: inter level migration flowchart.

Step 7. Run multi-level optimization procedure.

Step 8. Give optimal design (optimal and robust solution at lower cost).

The application of the proposed methodology requires the following:

- At element-level: the design parameters considered are geometric (section, thickness,...) which are specific to the element or sub-structure.

- At system-level: in this step, the material properties of the structure: Young's modulus $(E)$, density $(\rho)$, etc. are design parameters that will be considered.

- For embedding between sub-structures: from outset conditions for connections are required as a constraint in local optimization problems.

To solve this multi-level problem, a combination of multi-level GAs is proposed (Fig. 4). The number of GAs chosen depends on the system being studied and the number of sub-systems that it has.

The optimization is managed by the GA_global (server), which simultaneously activates the AG_elements (terminals) to solve the element problems. They can communicate with each other if the elements (sub-structures) are connected to each other. Once the AG_elements converge, GA_global uses the values of the optimal design parameters of the sub-structures (from optimization at element-level) to make global optimization, while respecting the constraints imposed on the structure or sub-structures. The results of this global optimization allow the designer to make decisions during the preproject and project phases.

\subsection{Robust modal synthesis method with respect to structural modifications and uncertainties}

To further reduce the computational time without losing the accuracy of the solutions, a robust modal synthesis method for the structural modifications and uncertainties is proposed. This method, based on the KL approach, is used in the optimization procedure discussed in this paper.

If we attribute a level of uncertainty to either zones or sub-structures, the stochastic equilibrium equation of the damped non-linear structure submitted to a deterministic harmonic excitation, can be written as follows:

$$
\left(-\omega^{2} M(\theta)+(1+i \eta) K(\theta)\right) U(\omega, \theta)=f_{e}(\omega)
$$

or

$$
\left(Z_{0}(\theta)+\Delta Z(\omega, \theta)\right) U(\omega, \theta)=f_{e}(\omega)
$$

where the mean dynamic stiffness matrix is

$$
Z_{0}(\omega)=\left(-\omega^{2} M_{0}+(1+i \eta) K_{0}\right) U(\omega, \theta)
$$


The stochastic dynamic stiffness matrix is

$$
\Delta Z(\omega, \theta)=\sum_{r=1}^{q}\left(-\omega^{2} M_{r}+(1+i \eta) K_{r}\right) \xi_{r}
$$

$U(\omega, \theta)$ is the response vector of the stochastic model and $f_{e}(\omega)$ is the vector of the applied forces.

Eq. (10) can be rewritten in the form

$$
Z_{0}(\omega) U(\omega, \theta)=f_{\Delta}(\omega, \theta)+f_{e}(\omega)
$$

where $f_{\Delta}(\omega, \theta)=-\Delta Z(\omega, \theta) U(\omega, \theta)$ is the random force vector associated with the unknown modifications of the initial structure. Eq. (11) is then interpreted as the dynamic equilibrium equation of the non-linear initial model submitted to $f_{4}(\omega, \theta)$.

In practice, the resolution of the non-linear problem (Eq. (11)) by the Monte Carlo simulation is very expensive. The condensation of this model by the standard reduction method is insufficiently predictive and robust with respect to the parametric perturbations. Thus we can then develop a dynamic condensation method adapted to the stochastic models [15] which uses an iterative procedure. The stochastic model condensed by a reduction of the nominal model, $T_{0}$ can be written, in the frequency domain, as follows:

$$
Z_{0}^{c}(\omega) U^{c}(\omega, \theta)=f_{\Delta}^{c}(\omega, \theta)+f_{e}^{c}(\omega)
$$

The optimal modes are extracted from the frequency responses sampled from each sub-structure submitted to the junction forces at the interfaces. These modes $\left(\phi_{1}, \ldots, \phi_{k}, \ldots, \phi_{M}\right)$ are determined by using the "snapshots" method in the frequency domain, where one can suppose that $\phi_{k}(x)(k=1, \ldots, M)$ is a linear combination of samples

$$
\phi_{k}=\sum_{i=1}^{M} \alpha_{i, k} U_{i}
$$

where $\alpha_{i, k}$ are the eigenvectors of the problem: $C A_{k}=\lambda_{k} A_{k}$ with $k=1, \ldots, M$; and $C$ is the covariance matrix with a general term

$$
C_{i j}=\frac{1}{M} U_{i}^{T} U_{j}(i, j=1, \ldots, M) \text { and } A_{k}=\left[\begin{array}{lll}
\alpha_{1, k} & \ldots & \alpha_{M, k}
\end{array}\right]^{T}
$$

The responses $U_{i}(i=1, \ldots, M)$ which intervene in Eq. (13) are obtained from the movement equation of a sub-structure submitted to the only junctions forces $F_{j}$

$$
\left(K_{0}-\omega^{2} M_{0}\right) U(\omega)=F_{j}(\omega)
$$

These forces being a priori unknown, the proposed method in [15] uses the "single composite input" technique to excite the sub-structure at the level of the $j$ degrees-of-freedom (DOF) of junctions.

Thus, at each frequency, $\omega_{k}$, one can introduce an excitation force $F_{j}(\omega)=\left[\begin{array}{ll}F_{j}\left(\omega_{k}\right) & 0\end{array}\right]^{T}$ in the form

$$
F_{j}\left(\omega_{k}\right)=\left[\left|\frac{1}{i \omega_{k}-i \Omega_{1}}\right|\left|\frac{1}{i \omega_{k}-i \Omega_{2}}\right| \cdots\left|\frac{1}{i \omega_{k}-i \Omega_{j}}\right|\right]^{T}
$$

where $i^{2}=-1$. The frequencies $\Omega_{k}(k=1,2, \ldots, j)$ are chosen arbitrarily in the sampling range $[0, \Omega]$.

The modes $\phi_{k}$ are used as a condensation basis and the transformation $K L$ takes the following form:

$$
T_{K L}=\left[\begin{array}{lll}
\phi_{1} & \ldots & \phi_{k}
\end{array}\right], \quad k \leq M
$$

The enrichment of the basis $T_{K L}$ by the random static residual uses the $M$ responses, $U_{0_{1}}, \ldots, U_{0_{M}}$. For each pulsation $\omega_{k}$ $(k=1, \ldots, M)$, the force vector of the uncertain parametric modification is written as

$$
f_{\Delta_{k}}=-\left(\Delta K-\omega_{k}^{2} \Delta M\right) U_{0}\left(\omega_{k}\right)
$$

The set of vectors, $f_{A_{k}}$ allows the generation of the force basis, $F_{\Delta}$ and the static residual matrix, $R_{\Delta}=K^{-1} F_{\Delta}$ associated with the stochastic modification forces allows the enriched optimal basis of KL to be constructed

$$
\mathrm{T}=\left[\begin{array}{lll}
\mathrm{T}_{\mathrm{KL}} & \mathrm{R}_{\Delta}
\end{array}\right]
$$

Finally, we can dispose of the condensed stochastic model to calculate the response of the stochastic structure.

\section{Numerical simulations}

\subsection{Two-dimensional frame}

We studied a two-dimensional frame (Fig. 5) [16], composed of three columns of section "I" and 12 horizontal beams of section "U" (Fig. 6). The horizontal beams are welded to the columns. The mechanical properties and geometric 
characteristics of the structure are:

- The structure is homogeneous: Young's modulus $E=2.110^{11} \mathrm{~N} / \mathrm{m}^{2}$ and density $\rho=7800 \mathrm{~kg} / \mathrm{m}^{3}$.

- The lengths of columns and horizontal beams are, respectively, 6 and $3 \mathrm{~m}$.

- The geometric characteristics of columns (Fig. 6) are

$$
a_{1}=42 \mathrm{~mm} ; \quad a_{2}=70 \mathrm{~mm} ; \quad a_{3}=6 \mathrm{~mm} ; \quad a_{4}=4 \mathrm{~mm} .
$$

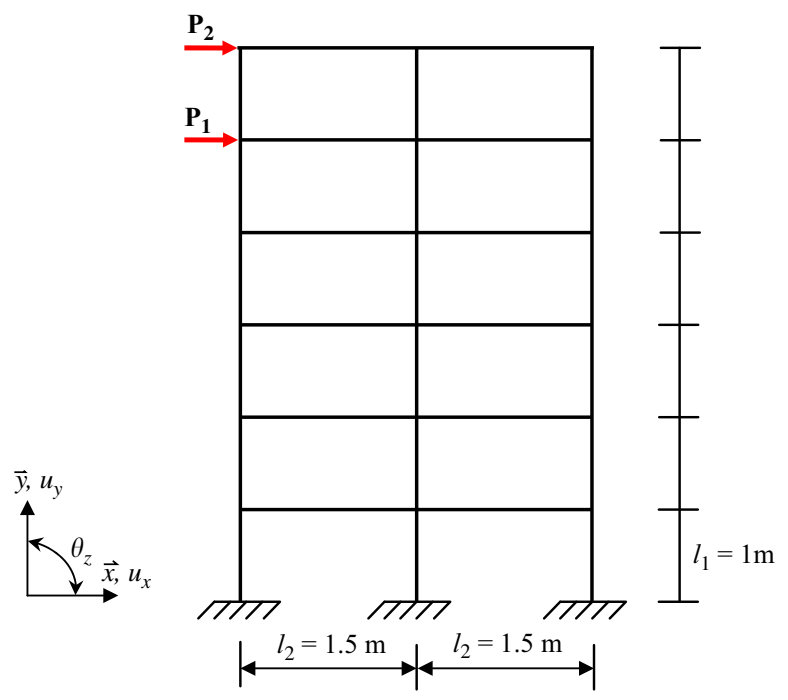

Fig. 5. Two-dimensional frame.
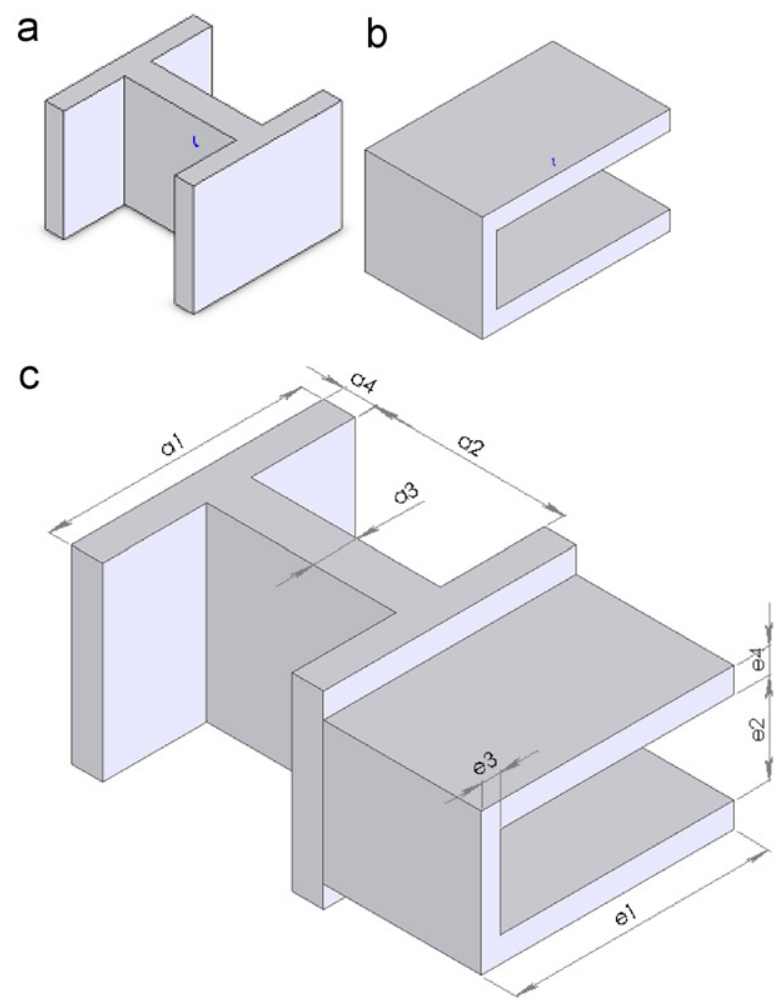

$\mathrm{a}_{1}=\mathrm{e}_{1}$

Fig. 6. Section of two-dimensional frame. 
- The geometric characteristics of horizontal beams (Fig. 6) are

$$
e_{1}=42 \mathrm{~mm} ; \quad e_{2}=56 \mathrm{~mm} ; \quad e_{3}=5 \mathrm{~mm} ; \quad e_{4}=8 \mathrm{~mm} .
$$

The FE model of the overall structure has 54 DOF. The frame structure is supported with fixed ends at nodes 1,2 and 3 (Fig. 5). The structure is excited at nodes 16 and 19 as: $\vec{P}_{1}=P_{1} \cos \omega t \cdot \vec{x}$ and $\vec{P}_{2}=P_{2} \cos w t . \vec{x}$, where $P_{1}=P_{2}=1 N$. In this example, we solve the dynamic problem

$$
M \ddot{y}(t)+C \dot{y}(t)+K y(t)=P(t)
$$

where $M, C$ and $K$ are, respectively, the mass matrix, damping and stiffness of the structure. And $y$ and $P$ are, respectively, the response of the model and the harmonic applied force.

The harmonic response calculated at node 19 from Eq. (20) is

$$
y(\omega)=\left(K-\omega^{2} M+j \omega C\right)^{-1} P
$$

Damping (proportional type) can be expressed in terms of stiffness by the relationship, $C=(\eta / \omega) K$, where $\eta \approx 0.02$.

The specification requires us to simultaneously minimize the overall mass and the maximum displacement at node 19 (denoted $\mathrm{MaxD}_{19}$ ) of the overall structure.

To meet the requirements of this specification, we divided the global structure into two groups of sub-structures. The first group is composed by columns of section "I" while the second is formed by horizontal beams of section " $U$ ". Fig. 7 illustrates the methodology of decomposition into sub-structures (levels).

The element-level RMO problem is broken into two groups of elements: the columns and horizontal beams (Eq. (21)).

First group of elements Second group of elements

$$
\left\{\begin{array} { l } 
{ \operatorname { m i n } ( m _ { i _ { i \in 1 , 2 , 3 } } , f _ { m _ { i } } ^ { v } ) } \\
{ x \in [ a _ { 1 } , a _ { 2 } , a _ { 3 } , a _ { 4 } ] } \\
{ \text { s.c } : \sigma _ { f } \leq \sigma _ { f _ { \operatorname { m a x } } } \mathrm { MPa } }
\end{array} \quad \left\{\begin{array}{l}
\min \left(m_{j_{j \in 1, \ldots 12}}, f_{m_{j}}^{v}\right) \\
x \in\left[e_{1}, e_{2}, e_{3}, e_{4}\right] \\
\text { s.c }: \sigma_{c} \leq \sigma_{c_{\max }} \mathrm{MPa}
\end{array}\right.\right.
$$

While the system-level RMO problem is (Eq. (22))

$$
\left\{\begin{array}{l}
\min \left(M_{g}, f_{M_{g}}^{v}, \operatorname{Max} D_{19}, f_{\operatorname{Max} D_{19}}^{v}\right) \\
Y \in\left[\rho, E, a_{1}^{*}, a_{2}^{*}, a_{3}^{*}, a_{4}^{*}, e_{1}^{*}, e_{2}^{*}, e_{3}^{*}, e_{4}^{*}\right] \\
\text { s.c }: a_{1}^{*}=e_{1}^{*}
\end{array}\right.
$$

where $M_{g}, \operatorname{Max} D_{19}, f_{M_{g}}^{v}$ and $f_{\mathrm{Max}_{19}}^{v}$ are the global mass of the structure, the maximum displacement at node 19 and their vulnerabilities, respectively, And (*) indicates that the design parameters are optimized at element-level. It should be noted that all the design parameters to be taken at system- or element-level are random and follow a normal distribution. There is a $20 \%$ level of uncertainty for each design parameter.

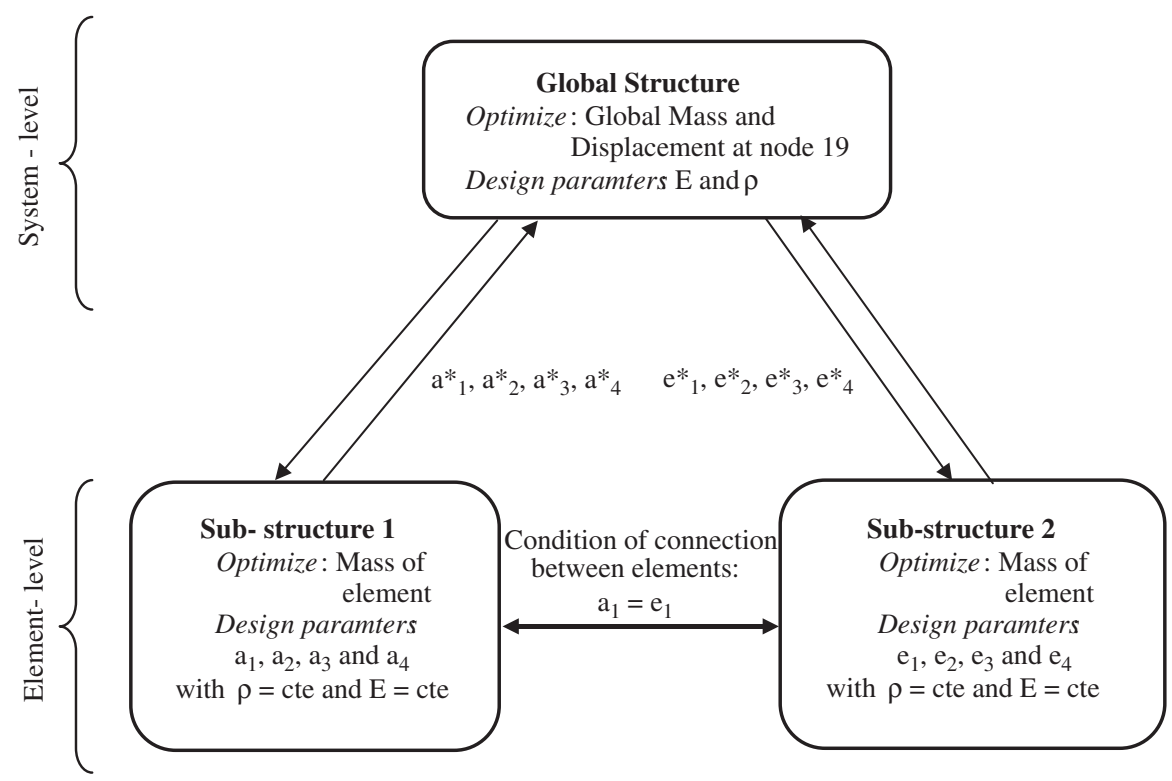

Fig. 7. Procedure of multi-level optimization of two-dimensional frame. 

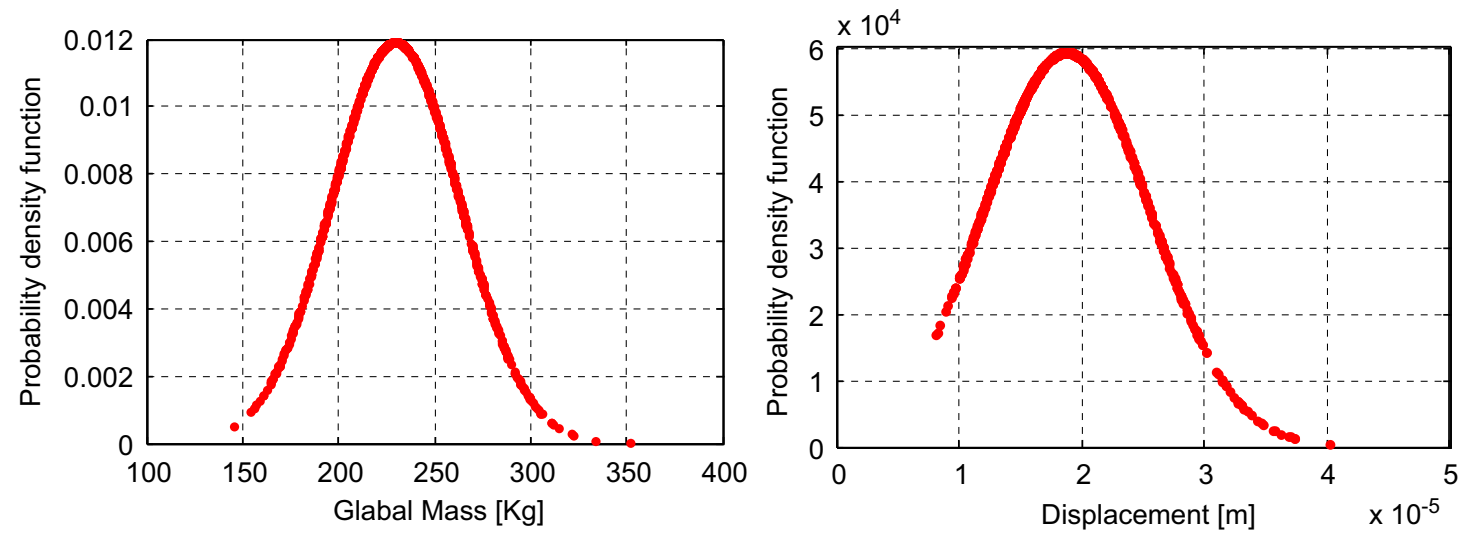

Fig. 8. Probability density function of the global mass and displacement obtained by Latin Hypercube method.

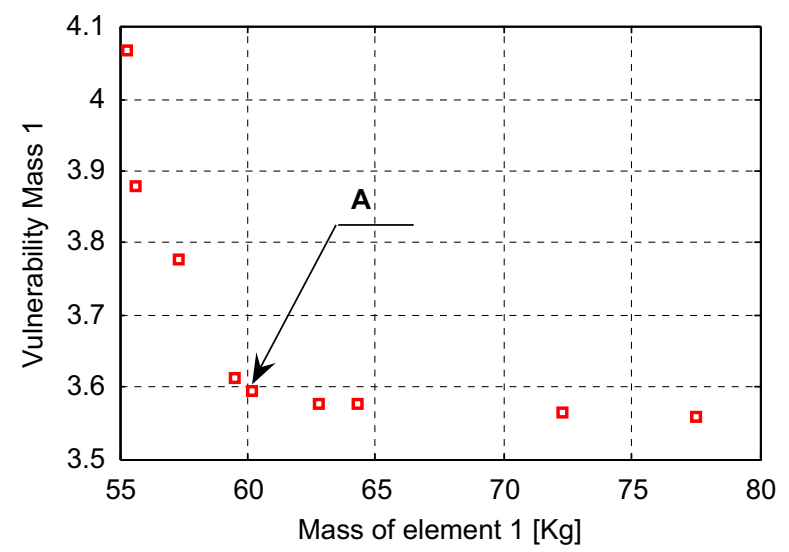

Fig. 9. Whole of robust Pareto optimal solutions: 1st group of elements.

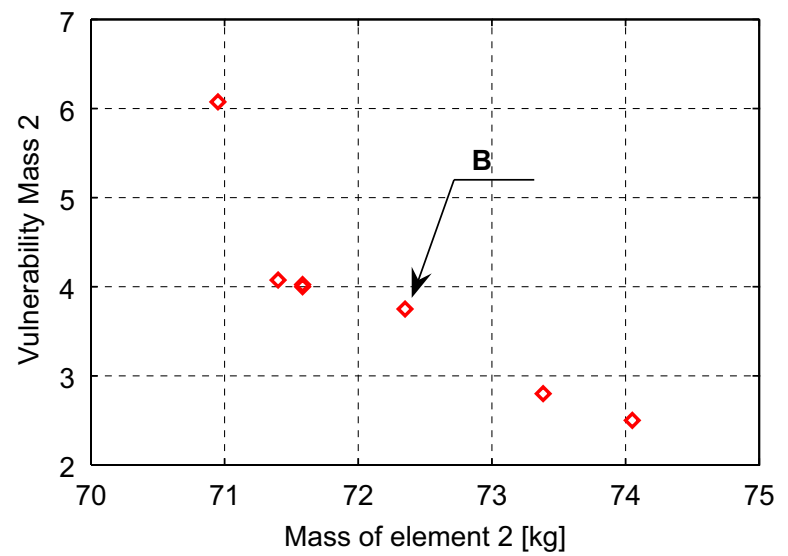

Fig. 10. Whole of robust Pareto optimal solutions: 2nd group of elements.

Fig. 8 shows the probability density function (pdf) of the global mass and displacement obtained by the Latin Hypercube method.

Solving the RMO problem for this first group of elements leads to a small number of solutions (Fig. 9). This is mainly due to the presence of a constraint function and vulnerability. We chose the solution that has the best compromise between mass and robustness (solution A). For the same reasons as given above, the resolution of the RMO problem for the second group of elements has led to a small number of solutions (Fig. 10). Similarly, a solution which was a good compromise between mass and robustness (solution B) was chosen. Taking account of the results of the RMO at 
element-level, the RMO problem at system-level is

$$
\left\{\begin{array}{l}
\min \left(M_{g}(Y), f_{M_{g}}^{v}(Y), \operatorname{Max} D_{19}(Y), f_{\text {MaxD }}^{v}(Y)\right) \\
Y \in\left[\rho, E, a_{19}^{*}, a_{2}^{*}, a_{3}^{*}, a_{4}^{*}, e_{1}^{*}, e_{2}^{*}, e_{3}^{*}, e_{4}^{*}\right] \\
\text { with : } a_{1}^{*}=e_{1}^{*}
\end{array}\right.
$$

The problem has four cost functions, two random design parameters and eight parameters which are fixed to their optimal levels from the element-level.

Fig. 11 presents all the Pareto optimal solutions in the space of cost functions. Fig. 12 shows the behavior of the overall structure before and after RMOL for solution C. Fig. 12 shows that the implementation of the proposed optimization procedure was able to lower the resonance peak of the first three modes in the frequency band $(0-100 \mathrm{~Hz})$ and especially that of the second mode (the aim of our study) to $\Delta D \approx 90 \%$. A frequency shift was recorded for all the modes.

To determine the performance (CPU + precision) of the RMOL, it was compared with the classical optimization method (RMO method applied to global system). The single-level RMO problem to be solved has four cost functions and nine random design parameters

$$
\left\{\begin{array}{l}
\min \left(M_{g}(Y), f_{M_{g}}^{v}(Y), \operatorname{Max} D_{19}(Y), f_{\mathrm{MaxD} D_{19}}^{v}(Y)\right) \\
Y \in\left[\rho, E, a_{1}, a_{2}, a_{3}, a_{4}, e_{1}, e_{2}, e_{3}, e_{4}\right] \\
\text { with }: a_{1}=e_{1}
\end{array}\right.
$$
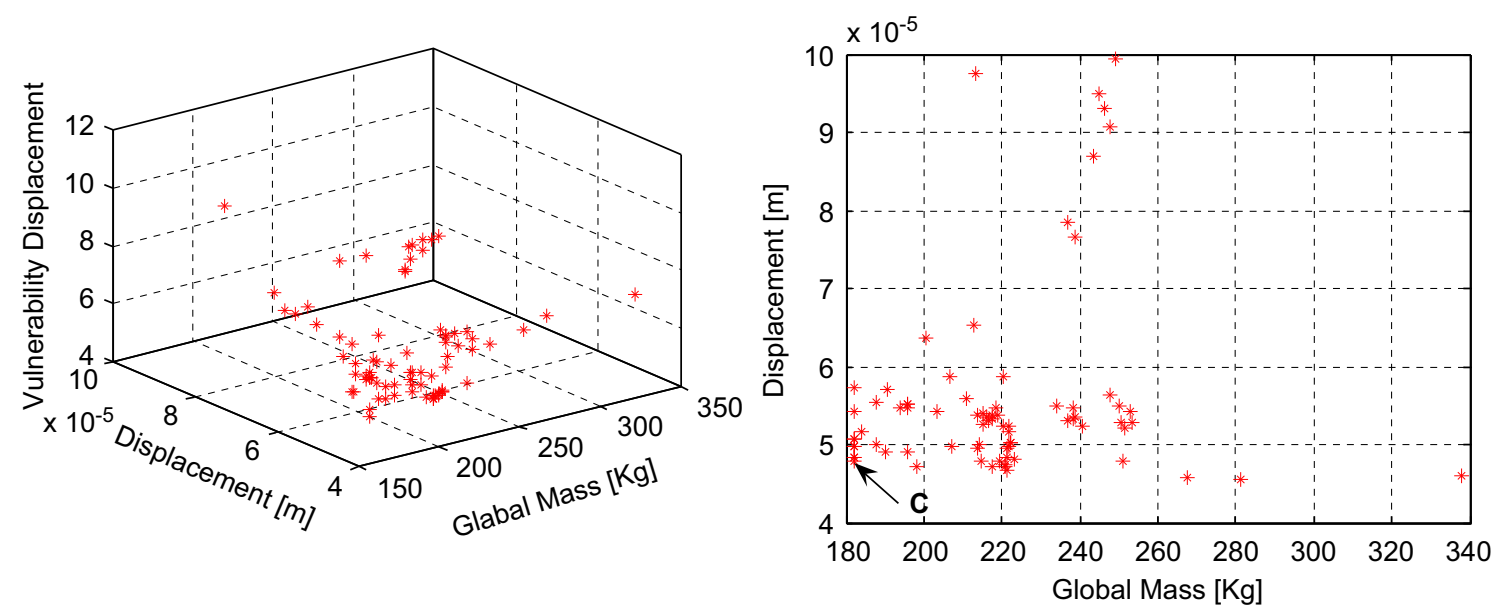

Fig. 11. (a) and (b): Whole robust Pareto optimal solutions: system-level.

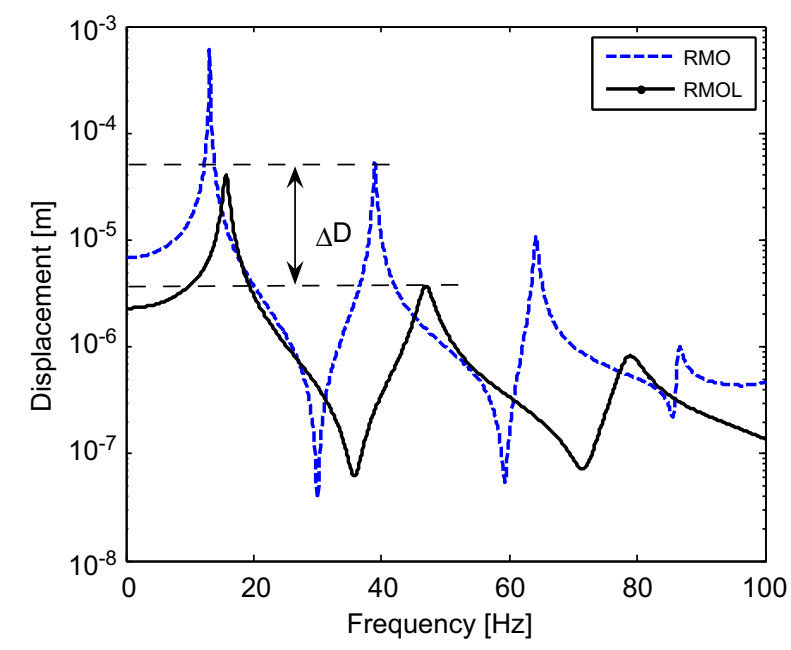

Fig. 12. Behavior of the structure before and after RMOL: Solution C. 


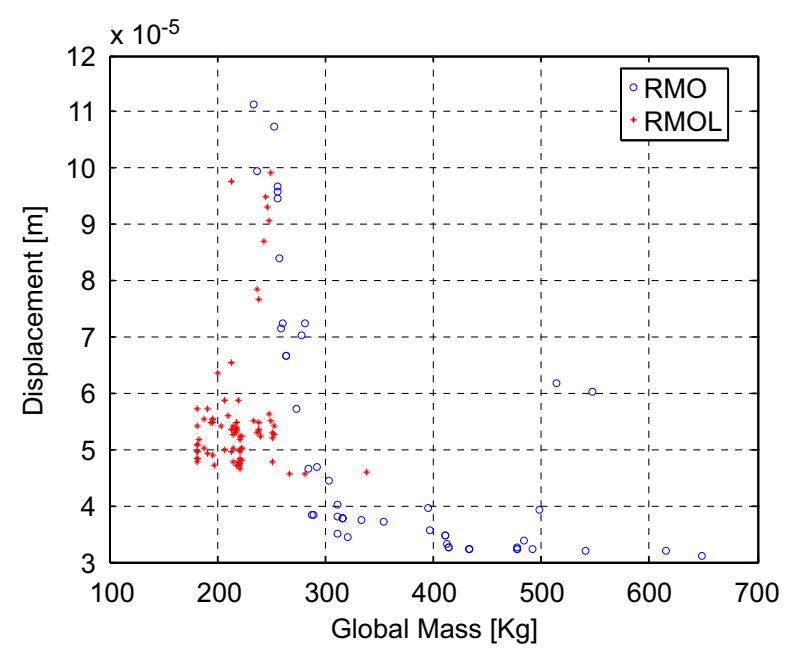

Fig. 13. Whole of robust Pareto optimal solutions: RMO and RMOL.

Table 2

Performance of the proposed method.

\begin{tabular}{llll}
\hline & CPU (\%) & Reduction (\%) & Error (\%) \\
\hline RMO & 100 & 30 & 6 \\
RMOL & 70 & & \\
\hline
\end{tabular}

Fig. 13 presents all the Pareto optimal and robust solutions as cost functions for the two methods (single-level and multi-level). Table 2 presents the performance of the proposed method compared to single-level method. There is a significant reduction in CPU time by about $30 \%$ and a precision error of less than $6 \%$. Thus, the engineer is able to make a rapid decision with acceptable accuracy in the project or pre-project phases. It should be noted that the error is determined by the Mahalanobis distance ratio [17], calculated for each cloud solution for each method, compared to the reference. The latter is created by applying the single-level RMO method which has undergone a $1 \%$ change.

\subsection{GARTEUR SM-AG 19 structure}

The proposed example (Fig. 14), concerns the Group for Aeronautical Research and Technology in Europe (GARTEUR) airplane model [18]. It is a simplified model of an aircraft (length $1.5 \mathrm{~m}$ and wide $2 \mathrm{~m}$ ), with a fuselage, wings and a tail [19] (Table 3). The FE model has 91 elements and a total of 906 DOFs. Free boundary conditions are assumed. Fig. 15 shows the simulated four-mode shapes used by GARTEUR. Fig. 16 gives the excitation nodes $(1,2, \ldots, 11)$ and the observation node located at point 1. The RMOL concept is shown in Fig. 17. This method uses a set of interconnected GAs. It should be noted that the process of multi-level optimization is preceded by a reduction in the FE model used for the structure and sub-structures.

The structure (system-level) is divided into five sub-structures (element-level) (see Fig. 14). In this case, five RMO problems are solved (Eq. (25))

$$
\left\{\begin{array} { l } 
{ \operatorname { m i n } ( m _ { 1 } , f _ { m _ { 1 } } ^ { v } ) } \\
{ x \in [ h _ { 1 } , L _ { 1 } , l _ { 1 } , L _ { 1 2 } ] , } \\
{ \text { s.c } : D _ { 1 } \leq D _ { 1 _ { \mathrm { Max } } } }
\end{array} \quad \left\{\begin{array}{l}
\min \left(m_{2}, f_{m_{2}}^{v}\right) \\
x \in\left[h_{2}, L_{2}, l_{2}\right] \\
\text { s.c }: D_{2} \leq D_{2_{\mathrm{Max}}}
\end{array},\left\{\begin{array}{l}
\min \left(m_{3}, f_{m_{3}}^{v}\right) \\
x \in\left[h_{3}, L_{3}, l_{3}\right] \\
\text { s.c }: D_{3} \leq D_{3_{\mathrm{Max}}}
\end{array},\left\{\begin{array}{l}
\min \left(m_{4}, f_{m_{4}}^{v}\right) \\
x \in\left[h_{4}, L_{4}, l_{4}\right] \\
s . c: D_{4} \leq D_{4_{\operatorname{Max}}}
\end{array},\left\{\begin{array}{l}
\min \left(m_{5}, f_{m_{5}}^{v}\right) \\
x \in\left[h_{5}, L_{5}, l_{5}\right] \\
\text { s.c }: D_{5} \leq D_{5_{\mathrm{Max}}}
\end{array}\right.\right.\right.\right.\right.
$$

where $m_{i / i=1, \ldots, 5}$ and $f_{m_{i / i=1, \ldots .5}}^{v}$ are, respectively, the mass and its vulnerability at each sub-structure. $D_{1_{\text {Max }}}, D_{2_{\text {Max }}}, D_{3_{\text {Max }}}, D_{4_{\text {Max }}}$ and $D_{5_{\operatorname{Max}}}$ are local constraint functions.

While the robust multi-objective problem at system-level is

$$
\left\{\begin{array}{l}
\min \left(M_{g}(Y), f_{M_{g}}^{v}(Y), U(Y), f_{U}^{v}(Y)\right) \\
Y \in\left[E_{1}, \rho_{1}, E_{2}, \rho_{2}, E_{3}, \rho_{3}, E_{4}, \rho_{4}, E_{5}, \rho_{5}, h_{1}^{*}, h_{2}^{*}, h_{3}^{*}, h_{4}^{*}, h_{5}^{*}, L_{1}^{*}, L_{2}^{*}, L_{3}^{*}, L_{4}^{*}, L_{5}^{*}, l_{1}^{*}, l_{2}^{*}, l_{3}^{*}, l_{4}^{*}, l_{5}^{*}\right]
\end{array}\right.
$$

where $M_{g}, f_{M_{g}}^{v}, U$ and $f_{U}^{v}$ are, respectively, the global mass of the structure, its vulnerability, the displacement at node (1) and its vulnerability, $(*)$ indicates that the design parameters are optimized at element-level.

It should be noted that all the design parameters to be taken at the system- or element-level are random and follow a normal distribution, and the level of uncertainty for each design parameter is $20 \%$. 


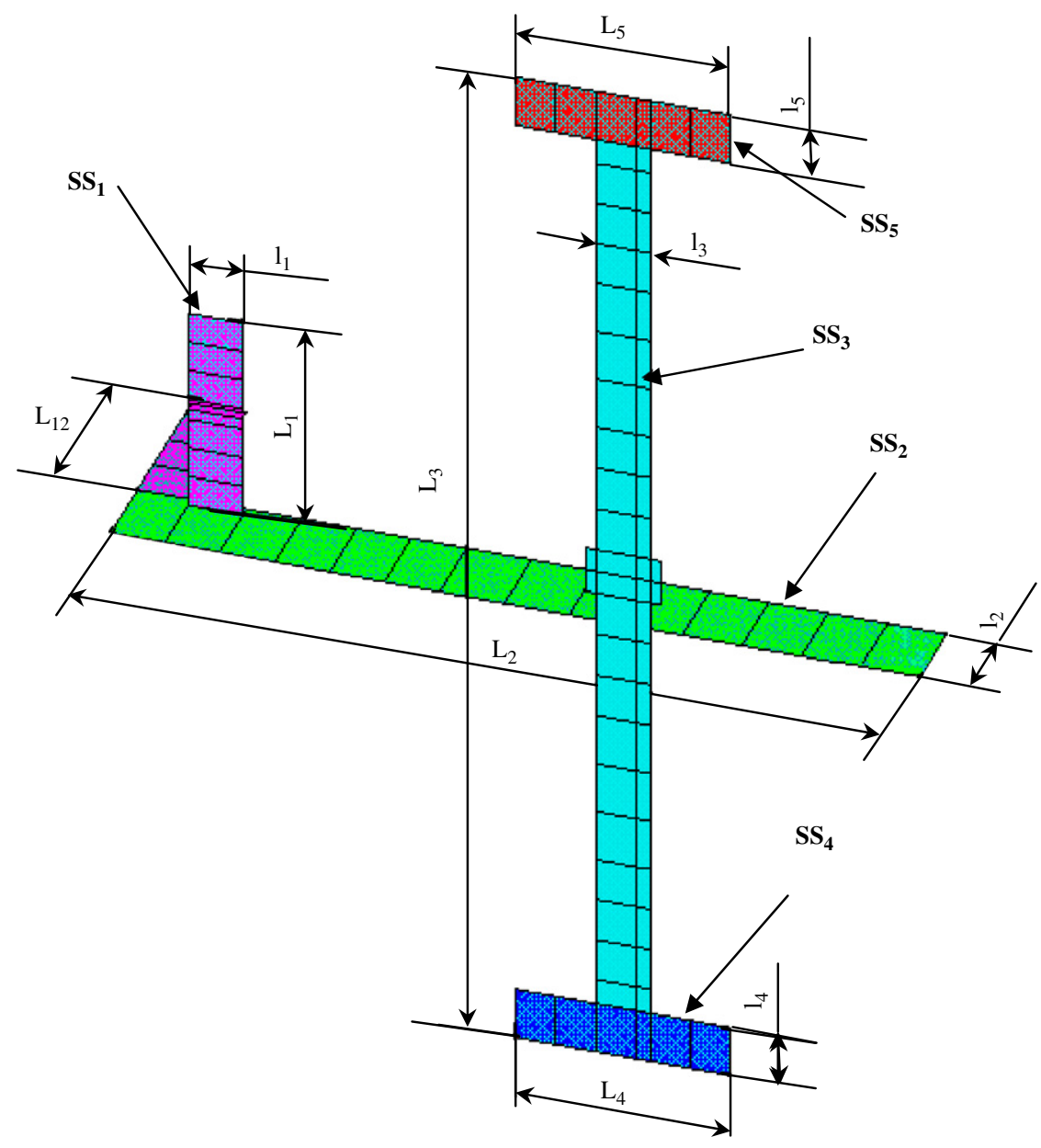

Fig. 14. FE model of the GARTEUR.

Fig. 18 shows the pdf of the global mass and displacement obtained by the Latin Hypercube method.

The resolution of this optimization problem (Eq. (26)) is preceded by a robust reduction, using the FE model of the structure by the KL method.

Fig. 19 illustrates the relative error curves on the eigenfrequencies and eigenvectors in the frequency range $(0-230 \mathrm{~Hz})$ (for the global structure case (GARTEUR)): The first 20 eigen modes calculated from the model condensed by the KL method are compared to those of the reference model. As shown in Fig. 19, the reduced model allows us to obtain a good frequency representation over the entire useful region of the band. The quality of the reduced model relative to the reference is also highlighted by the difference curve in terms of displacement (Fig. 20).

To compare the performance of the proposed RMOL method with the single-level RMO method, we propose to resolve the RMO method (without multi-level decomposition and reduction by FE model).

The single-level RMO problem to be solved has four cost functions and 25 random design parameters

$$
\left\{\begin{array}{l}
\min \left(M_{g}(Y), f_{M_{g}}^{v}(Y), D(Y), f_{D}^{v}(Y)\right) \\
Y \in\left[E_{1}, E_{2}, E_{3}, E_{4}, E_{5}, \rho_{1}, \rho_{2}, \rho_{3}, \rho_{4}, \rho_{5}, h_{1}, h_{2}, h_{3}, h_{4}, h_{5}, L_{1}, L_{2}, L_{3}, L_{4}, L_{5}, l_{1}, l_{2}, l_{3}, l_{4}, l_{5}\right]
\end{array}\right.
$$

Fig. 21 presents the whole Pareto optimal and robust solutions to the cost functions of the two methods (single-level RMO and RMOL). There is good agreement with the cloud of solutions from both methods.

Table 4 shows the performance of the proposed method compared to the single-level method. There is a reduction in CPU time of about $80 \%$ and a precision error of less than $3 \%$. This demonstrates the potential of the proposed methodology at the pre-project or project stages.

To compare the behavior of the structure before and after RMOL, we selected a solution to whole Pareto optimal and robust solutions. Solution A was selected from the area that represents the correct compromise between the cost functions which were considered. Fig. 22 shows the before and after behavior of the structure for solution A. 


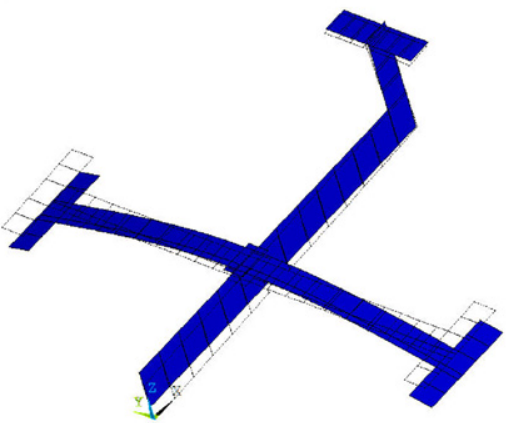

Mode $1: 7.039 \mathrm{~Hz}$

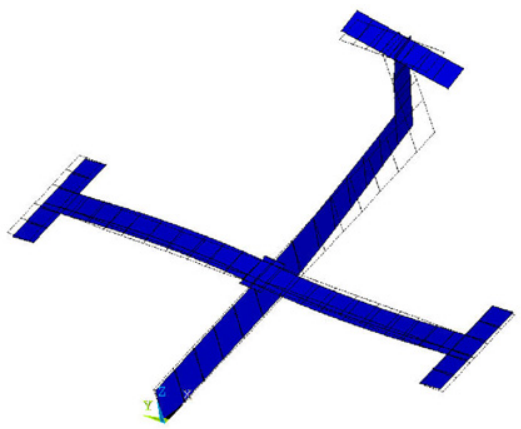

Mode $3: 17.223 \mathrm{~Hz}$

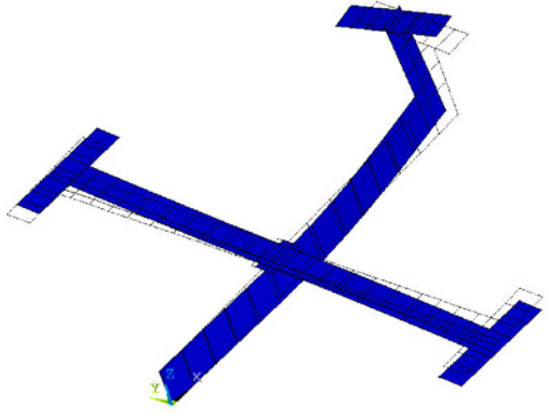

Mode $2: 7.934 \mathrm{~Hz}$

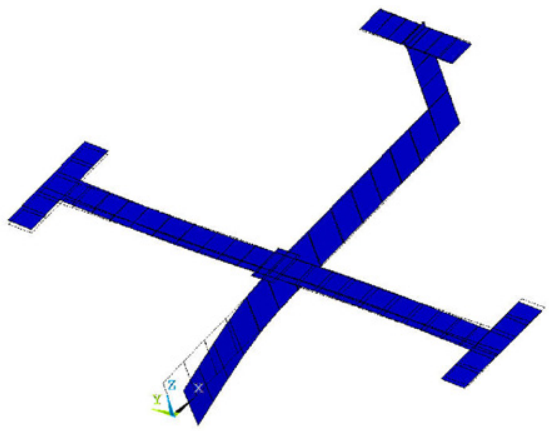

Mode 4 : $24.901 \mathrm{~Hz}$

Fig. 15. First four eigenmodes of GARTEUR.

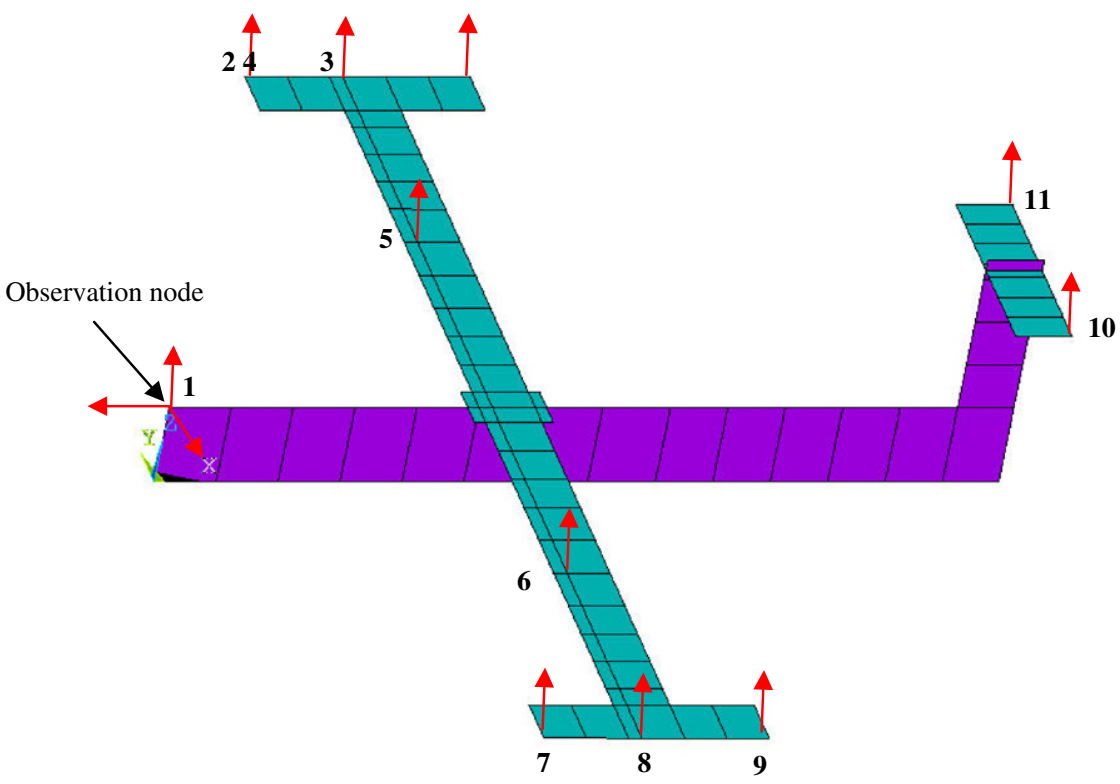

Fig. 16. Excitation and observation nodes.

\section{Conclusions}

We have proposed a RMOL method for the design of complex systems which have a large FE model. This method uses of a combination of interconnected GAs and a robust reduction of large FEM mechanical structures using the KL method.

The proposed method also utilizes the task sharing commonly used in structural engineering. In addition, it takes into account the presence of uncertainties in the design parameters during the optimization, which allows us to obtain robust optimal solutions. 


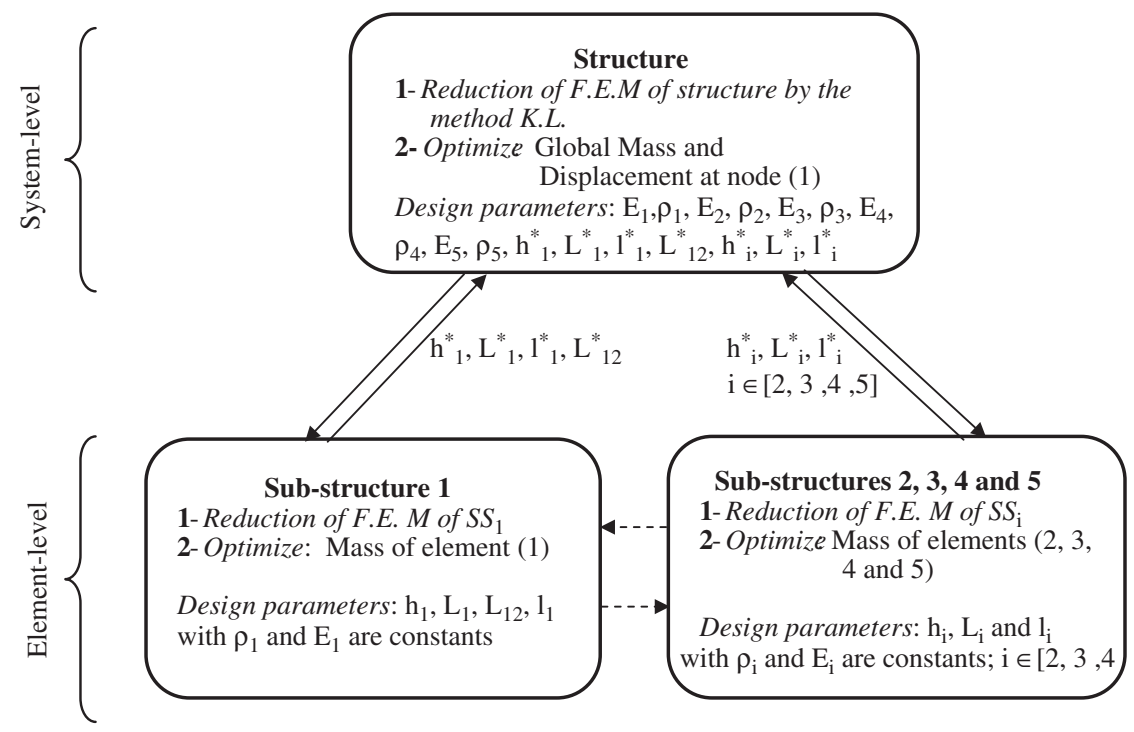

Fig. 17. Procedure of multi-level optimization of GARTEUR.
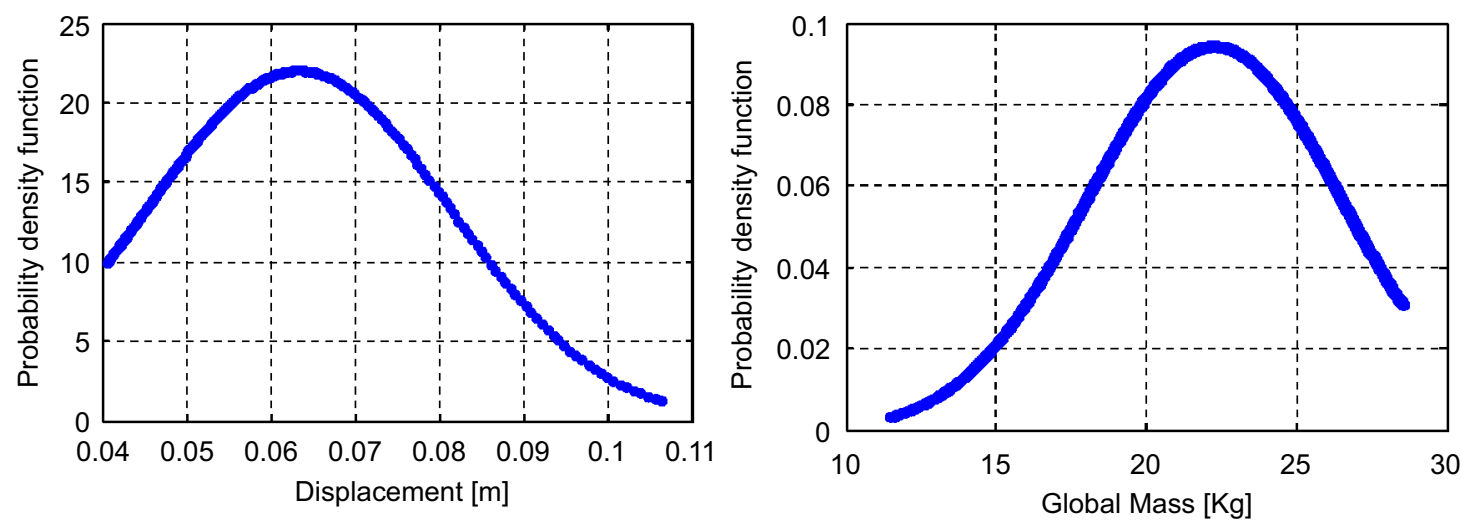

Fig. 18. Probability density function of the global mass and displacement obtained by Latin Hypercube method.

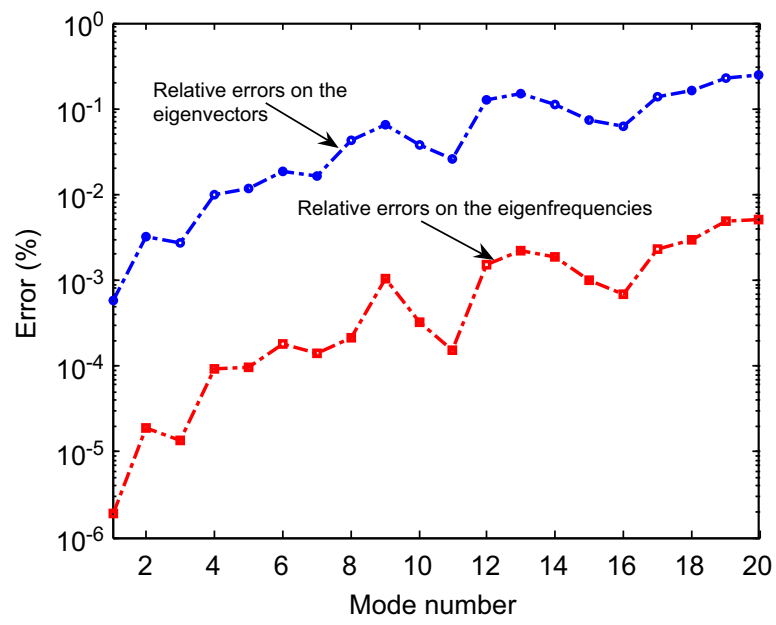

Fig. 19. Errors on the eigensolution in the frequency range [0-230 Hz] of GARTEUR. 


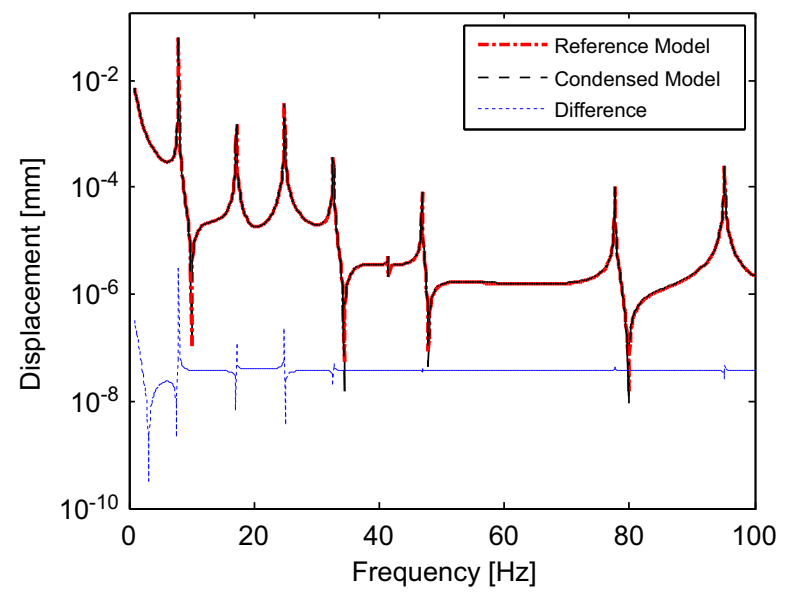

Fig. 20. Quality of the prediction of the condensed model by KL method at observation point.
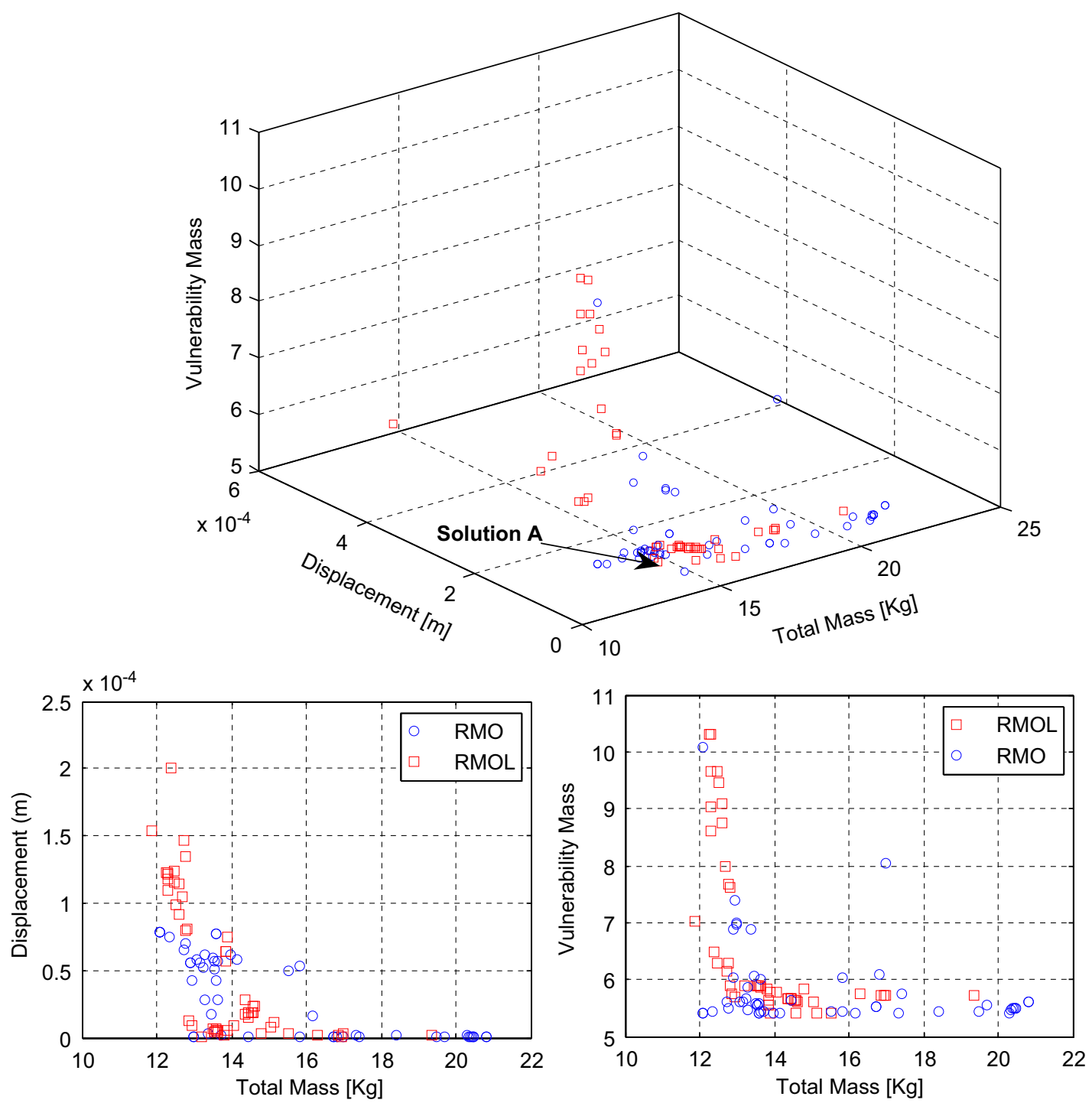

Fig. 21. Whole Pareto optimal and robust solutions. 
Table 3

Mean values of geometric and mechanical parameters of the structure.

\begin{tabular}{|c|c|c|c|c|c|}
\hline & $\mathbf{S S}_{1}$ & $\mathbf{S S}_{2}$ & $\mathbf{S S}_{3}$ & $\mathbf{S S}_{4}$ & $\mathbf{S S}_{\mathbf{5}}$ \\
\hline Geometric parameters (mm) & $\begin{array}{l}L_{1}=400 \\
l_{1}=100 \\
h_{1}=10 \\
L_{12}=300\end{array}$ & $\begin{array}{l}L_{2}=1500 \\
l_{2}=150 \\
h_{2}=50\end{array}$ & $\begin{array}{l}L_{3}=2000 \\
l_{3}=100 \\
h_{3}=10\end{array}$ & $\begin{array}{l}L_{4}=400 \\
l_{4}=100 \\
h_{4}=10\end{array}$ & $\begin{array}{l}L_{5}=400 \\
l_{5}=100 \\
h_{5}=10\end{array}$ \\
\hline Mechanical parameters $E_{i}\left(\mathrm{~N} / \mathrm{m}^{2}\right), \rho_{i}\left(\mathrm{Kg} / \mathrm{m}^{3}\right)$ & $\begin{array}{l}E_{1}=70.3 \times 10^{9} \\
\rho_{1}=1269\end{array}$ & $\begin{array}{l}E_{2}=70.3 \times 10^{9} \\
\rho_{2}=1269\end{array}$ & $\begin{array}{l}E_{3}=70.3 \times 10^{9} \\
\rho_{3}=1269\end{array}$ & $\begin{array}{l}E_{4}=70.3 \times 10^{9} \\
\rho_{4}=1269\end{array}$ & $\begin{array}{l}E_{5}=70.3 \times 10^{9} \\
\rho_{5}=1269\end{array}$ \\
\hline
\end{tabular}

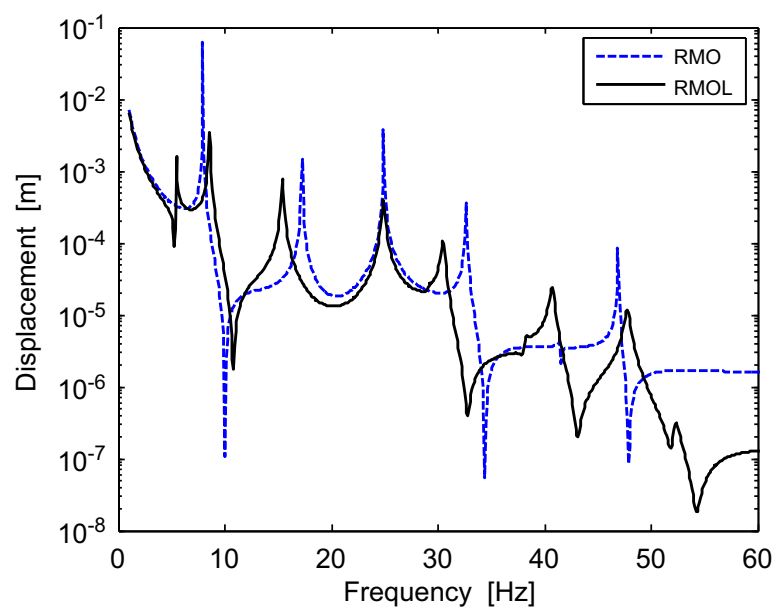

Fig. 22. Behavior of the structure before and after RMOL: Solution A.

Table 4

Performance of the proposed method.

\begin{tabular}{lccc}
\hline & CPU (\%) & Reduction (\%) & Error (\%) \\
\hline RMO & 100 & 80 & 3 \\
RMOL & 20 & & \\
\hline
\end{tabular}

The application of this method to mechanical structures like beams and plates has led to a drastic reduction in CPU computation time compared to the single-level optimization procedure. This indicates the potential of this method as powerful tool to aid decision-making during the pre-project or project steps when complex structures which need a large FE model are being designed.

\section{Appendix A}

The Karhunen-Loeve decomposition of the random field $H(\bullet)$ on the basis of the eigenfunctions $f_{r}(x)$ is given by

$$
\begin{aligned}
& H(x, \theta)=\mu(x)+\sum_{r=1}^{\infty} \sqrt{\lambda_{r}} f_{r}(x) \xi_{r}(\theta) \text { of truncated shape : } \\
& \hat{H}(x, \theta)=\mu(x)+\sum_{r=1}^{q} \sqrt{\lambda_{r}} f_{r}(x) \xi_{r}(\theta)
\end{aligned}
$$

where $\left\{\xi_{r}(\theta), r=1, \ldots, q\right\}$ are the independent Gaussian standard normal variables and $\left(\lambda_{r}, f_{r}\right)$ represent, respectively, the eigenvalues and eigenvectors of the known covariance function $C\left(x_{1}, x_{2}\right)$ associated to $H(\bullet)$, whose the spectral decomposition is written as

$$
C\left(x_{1}, x_{2}\right)=\sum_{r=1}^{\infty} \lambda_{r} f_{r}\left(x_{1}\right) f_{r}\left(x_{2}\right)
$$


The Stochastic mass Matrix is the assembly of the elementary matrices

$$
M(\theta)=\cup_{e}\left[\int_{\Omega_{e}} H(x, \theta) N^{T} N d \Omega_{e}\right] \approx M_{0}+\sum_{r=1}^{q} M_{r} \xi_{r}(\theta)
$$

with

$$
M_{0}=\cup_{e}\left[\int_{\Omega_{e}} \mu(x) N^{T} N d \Omega_{e}\right] \quad M_{r}=\cup_{e}\left[\int_{\Omega_{e}} \sqrt{\lambda_{r}} f_{r}(x) N^{T} N d \Omega_{e}\right]
$$

In the same way, the stochastic stiffness matrix is written as

$$
K(\theta) \approx K_{0}+\sum_{r=1}^{q} K_{r} \xi_{r}(\theta)
$$

with

$$
K_{0}=\cup_{e}\left[\int_{\Omega_{e}} \mu(x) B^{T} \mathrm{DB} d \Omega_{e}\right] ; \quad K_{r}=\cup_{e}\left[\int_{\Omega_{e}} \sqrt{\lambda_{r}} f_{r}(x) B^{T} \mathrm{DB} d \Omega_{e}\right]
$$

$\mathrm{D}$ is the matrix of elastic coefficients.

\section{References}

[1] Q.S. Li, D.K. Liu, J.Q. Fang, C.M. Tam, Multi-level optimal design of buildings with active control under winds using genetic algorithms, Journal of Wind Engineering and Industrial Aerodynamics 86 (2000) 65-86.

[2] L.U. Hansen, P. Horst, Multi-level optimization in aircraft structural design evaluation, Computers and Structures 86 (2008) $104-118$.

[3] G. Li, R.-G. Zhou, L. Duan, W.-F. Chen, Multiobjective and multilevel optimization for steel frames, Engineering Structures 21 (1999) 519-529.

[4] H. Engels, W. Becker, A. Morris, Implementation of a multi-level optimisation methodology within the e-design of a blended wing body, Aerospace Science and Technology 8 (2004) 145-153.

[5] A. Rothwell, Multi-level optimization of aircraft shell structures, Thin-Walled Structures II (1991) 85-103.

[6] Y. Ding, Multilevel optimization of frames with beam including buckling constraints, Computers \& Structures 32 (2) (1989) $249-261$.

[7] T. Norihiro, P.L. Daniel, Mu1ti-level optimization for multi-objective problems, Applied Mathematical Modelling 5 (1981).

[8] G. Masson, S. Cogan, N. Bouhaddi, J. Bonini, Parameterized reduced models for efficient optimization of structural dynamic behaviour, in: Proceedings of the 43rd AIAA/ASME/ASCE/AHS/ASC Structures, Structural Dynamics and Materials Conference, paper no 1392, Denver, 22-25, 2002.

[9] M. Guedri, N. Bouhaddi, R. Majed, Reduction of the stochastic finite element models using a robust dynamic condensation method, Journal of Sound and Vibration 297 (2006) 123-145

[10] K.H. Lee, I.S. Eom, G.J. Park, W.I. Lee, Robust design for unconstrained optimization problems using the Taguchi method, AIAA Journal 34 (1996) $1059-1063$.

[11] K.H. Lee, G.J. Park, Robust optimization considering tolerances of design variables, Computers and Structures 79 (2001) 77-86.

[12] B. Ait Brik, S. Ghanmi, N. Bouhaddi, S. Cogan, Robust design in structural mechanics, Engineering Science and Mechanics 8 (1) (2007) 33-43.

[13] R.L. Iman, W.J. Conver, Small sample sensitivity analysis techniques for computer models, with an application to risk assessment, Communications in Statistics A9 (1980) 1749-1842.

[14] N. Srinivas, K. Deb, Multi-objective Optimization using Nondominated Sorting in Genetic Algorithms, Technical Report, Department of Mechanical Engineering, Institute of Technology, India, 1993.

[15] T. Kim, Component mode synthesis method based on optimal modal analysis, in: Proceedings of the 43rd AIAA/ASME/ASCE/AHS/ASC Structures, Structural Dynamic and Materials Conference, Denver, AIAA Paper 2002-1226, 2002.

[16] S. Ghanmi, M. Guedri, M.-L. Bouazizi, N. Bouhaddi, Optimisation robuste multi-niveaux et multi-objectif, in: Proceedings of the $3^{\text {ième }}$ Congrès International Conception et Modélisation des Systèmes Mécaniques CMSM'2009, 16-18, Hammamet, Tunisie, 2009.

[17] S. Ghanmi, M.-L. Bouazizi, N. Bouhaddi, Robustness of mechanical systems against uncertainties, Finite Elements in Analysis and Design 43 (2007) 715-731.

[18] M. Degener, M. Hermes, Ground vibration test and finite element analysis of the GARTEUR SM-AG 19 testbed. Deutsche Forschungtalt fur Luft und Raumfahrt, Insititut für Aeroelastik, Goettingen, 1996.

[19] E. Balmès, J.R. Wright, GARTEUR Group on ground vibration testing results from the test of a single structure by 12 laboratories in Europe, in: Proceedings of DETC'97,1997 ASME Design Engineering Technical Conferences, September 14-17, Sacramento, California, 1997. 